\title{
riccafd
}

Revista Iberoamericana de Ciencias de la Actividad Física y el Deporte

\section{ANÁLISIS BIBLIOGRÁFICO DE LA GAMIFICACIÓN EN EDUCACIÓN FÍSICA}

\section{BIBLIOGRAPHICAL ANALYSIS OF GAMIFICATION IN PHYSICAL EDUCATION}

\author{
Juan Carlos Escaravajal Rodríguez ${ }^{1}$ y Fátima Martín-Acosta ${ }^{2}$ \\ 1 Juan Carlos Escaravajal Rodríguez. Facultad de Ciencias de la Educación, Universidad de \\ Granada, España. jcescaravajalrodriguez@gmail.com \\ ${ }^{2}$ Fátima Martín-Acosta. Fac. de Educación, Universidad de Cádiz, España. fatima.martin@uca.es \\ Correspondencia: Juan Carlos Escaravajal Rodríguez. Calle Ramón y Cajal, n 7A, Águilas \\ (Murcia), CP: 30880. Teléfono: 660684953. Email: jcescaravajalrodriguez@gmail.com
}

DOI: http://dx.doi.org/10.24310/riccafd.2019.v8i1.5770

\section{RESUMEN}

El objetivo del presente trabajo consiste en revisar, recopilar y analizar la bibliografía relacionada con la gamificación en el área de la Educación Física. Se realizó un revisión documental de los artículos publicados hasta septiembre de 2018 en las siguientes bases de datos: Dialnet, EBSCOhost, Google Scholar y Web of Science. Las palabras clave utilizadas fueron: gamificación, educación física, gamification y physical education. De esta búsqueda se obtuvieron un total de 93 artículos, posteriormente se llevo a cabo un proceso de selección final, obteniendo 19 trabajos. Los criterios de inclusión fueron: (1) artículos originales; (2) escritos en castellano o inglés; (3) que traten sobre cualquier aspecto de la gamificación en el área de Educación Física. En los resultados se observó que los primeros artículos de esta temática aparecieron en 2015 con presencia del $33,3 \%$ sobre total analizado. En relación a la tipología, principalmente son experiencias $(42,1 \%)$, seguidos de propuestas $(31,6 \%)$. Por último, las etapas educativas más presentes son la E.S.O. con un $52,2 \%$, seguida del ámbito universitario con un $26,1 \%$. En base a los resultados, se observan datos positivos, debido a que, posiblemente, cada vez más docentes e investigadores son más conscientes de los beneficios de la gamificación como herramienta educativa. Además, este trabajo es un útil recurso para profesionales interesados en esta temática, ya que pueden acceder de forma sencilla a todos los artículos existentes hasta el momento.

Palabras clave: revisión bibliográfica, metodologías activas, innovación, juego, rol. 


\section{ABSTRACT}

The aim of this project consists on reviewing, compiling and analyzing the bibliography incidental to the gamification in the area of Physical Education. A documentary review was conducted, including all the articles published until September 2018 in the following databases: Dialnet, EBSCOhost, Google Scholar and Web of Science. The keywords were: gamificación, Educación Física, gamification, Physical Education. A total of 93 articles were found, and after a selection procedure, we obtained 19 articles. The inclusion criteria were: (1) original articles; (2) written in either spanish or english; (3) articles dealing with anything related to the topic of gamification in Physical Education. In the results can be found that some of the first articles appeared in 2015, which represent a 33,3\% of the total. Related to the typology, they are mostly experiences $(42,1 \%)$, followed by proposals $(31,6 \%)$. Lastly, the most common educational stages are: E.S.O. $(52,2 \%)$, followed by the university area $(26,1 \%)$. According to these results some positive data can be seen due to the possibility that teachers and researchers are more aware of the gamification benefits as an educational tool. Furthermore, this project is useful for everyone interested in this topic, as it makes all articles related thus far easily accessible.

Key words: bibliographic review, active methodology, innovation, game, rol.

\section{INTRODUCCIÓN}

Como es sabido, la gamificación es una forma de ludificación del aprendizaje, de hacer del juego el centro del aprendizaje. Deterding, Khaled, Nacke y Dixon (2011) la definen como el uso de elementos del juego en contextos no lúdicos. Como apoyan diversos estudios, el juego ayuda a adquirir aprendizajes de forma significativa lo que permite que sean mejor interiorizados y consolidarlos en el tiempo a diferencia de los contenidos memorizados o descontextualizados. Además, es muy valorada como estrategia motivacional, tanto para la motivación intrínseca como extrínseca (Díaz y Troyano, 2013; Landers y Callan, 2011; Monguillot, González, Zurita, Almirall y Guitert, 2015; Ordiz, 2017).

Son ya muchos los ámbitos en los que se conoce y se lleva a cabo la gamificación, siendo la educación junto con el entretenimiento y recreación, las artes y la comunicación, los sectores a los que ha llegado antes a España, pues ya en 2012 se habla de un porcentaje muy elevado de conocimiento sobre la gamificación entre los profesionales de dichas áreas (Game Marketing, 2012).

Dentro de la educación el uso de la gamificación se ha extendido a todas las áreas y niveles. Actualmente es posible ver su aplicación en una gran variedad de contenidos en Educación Primaria como Matemáticas (Ortegón, 2016), Ciencias Sociales (Guevara, 2015), y Lengua y Literatura (Marí, 2015); en Educación Segundaria Obligatoria (E.S.O.) (Quintanal, 2016) y en el ámbito universitario 
(González y Mora, 2015; Pérez-López, Rivera y Trigueros, 2017). Además, existen diversas revisiones de la bibliografía sobre la gamificación en el espacio educativo (Johnson et al., 2016; Kasurinen y Knutas, 2016; Lozada-Ávila y Betancur, 2016; Sardi, Idri y Fernández-Alemán, 2017), pero ninguna que recoja trabajos existentes que traten de forma específica la gamificación en Educación Física (EF).

Centrándonos en el área de la EF, Fernández-Río, Calderón, Hortigüela, Pérez-Pueyo y Aznar (2016) presentan los modelos pedagógicos más utilizados, significativos y emergentes en esta área, y entre los cuales no se encuentra la gamificación. Sin embargo, otros autores la consideran a la misma vez una técnica, un método y una estrategia (Marín e Hierro, 2013). Encontrándose la gamificación en estado de consolidación como una estrategia de aprendizaje emergente para la educación actual por llevar implícita características positivas como favorecer la motivación e interés del alumnado por aprender, la mejora del rendimiento y un aumento del compromiso por la práctica de actividad física (Monguillot et al., 2015; Ordiz, 2017), tan reconocidas son sus posibilidades y beneficios educativos entre la comunidad de docentes de EF que, aunque son escasas, ya se pueden observar propuestas y experiencias en este ámbito (Mora-González y Martínez-Téllez, 2015; Navarro, Martínez y Pérez; 2017).

Por tanto, el objetivo del presente trabajo consiste en revisar, recopilar y analizar la bibliografía relacionada con la gamificación en el área de la Educación Física.

\section{METODOLOGÍA}

\section{Diseño}

El diseño utilizado para esta investigación ha sido la revisión documental descriptiva (Hernández, Fernández y Baptista, 2010) de la bibliografía científica relacionada con la gamificación en Educación Física.

\section{Procedimiento}

La búsqueda se centró en artículos publicados hasta septiembre de 2018, para ella se utilizaron las siguientes bases de datos: Dialnet, EBSCOhost, Google Scholar y WOS (Web of Science). Las palabras clave utilizadas fueron: gamificación, educación física, gamification y physical education. Los criterios de inclusión fueron: (1) artículos originales; (2) escritos en castellano o inglés; (3) que traten sobre cualquier aspecto de la gamificación en el área de Educación Física. Se excluyeron las comunicaciones a congresos, las tesis doctorales, y los trabajos fin de grado o máster. Tras aplicar esta estrategia se seleccionaron un total de 19 artículos (figura 1). 
Búsqueda en bases de datos: Dialnet, EBSCOhost, Google Scholar y WOS.

\begin{tabular}{|c|c|}
\hline $\begin{array}{l}\text { Resultados de la búsqueda: } 93 \\
\qquad \mid--------\end{array}$ & $\begin{array}{c}\text { Criterios de inclusión: } \\
\text { (1) artículos originales, } \\
\text { (2) escritos en castellano o inglés, } \\
\text { (3) que traten sobre cualquier aspecto de la } \\
\text { gamificación en el área de la Educación } \\
\text { Física. }\end{array}$ \\
\hline $\begin{array}{l}\text { Total de artículos incluidos en } \\
\text { la revisión: } 19\end{array}$ & $\begin{array}{l}\text { Se excluyeron las comunicaciones a } \\
\text { congresos, las tesis doctorales, y los } \\
\text { trabajos fin de grado o máster. }\end{array}$ \\
\hline
\end{tabular}

Figura 1. Proceso de selección de los artículos.

\section{Análisis estadístico}

Para el análisis de los datos obtenidos se realizó una estadística descriptiva con el software Statistical Package for Social Sciences v.20.0 (SPSS).

\section{RESULTADOS}

El porcentaje predominante de artículos publicados según la etapa educativa a la que estaban enfocados fue el $52,2 \%$ para la E.S.O, seguido por el $26,1 \%$ en la universidad y con valores inferiores en Bachillerato (13\%) y Primaria $(8,7 \%)$. Las primeras publicaciones obtenidas fueron a partir de 2015 , año en el cual se encontraron el mayor número de ellas (33,3\%). En 2016 es el año con menor porcentaje (9.5\%), volviendo a aumentar en los siguientes años hasta la actualidad con los mismos porcentajes, $28.6 \%$ tanto para 2017 como 2018. Atendiendo a la tipología, los artículos fueron clasificados en "propuesta", "experiencia" e "investigación". Los trabajos en los se muestran propuestas que fueron llevadas a cabo son las más numerosas $(42,1 \%)$ frente a las que no fueron desarrolladas $(31,6 \%)$ o eran trabajos de investigación $(26,3 \%)$.

Para analizar los artículos, éstos se clasifican según la etapa en la que están enfocados. De esta manera, se encuentra un grupo que engloba aquellos enfocados en Educación Primaria (tabla 1), otro con enfocados en Educación Secundaria Obligatoria y Bachillerato (tabla 2), y por último, enfocados en la etapa universitaria (tabla 3). 
Tabla 1: Trabajos enfocados en Primaria.

\begin{tabular}{llll}
\hline Autoría & Tipo* & Curso & Resultados y/o conclusiones \\
\hline $\begin{array}{l}\text { Fernández, Prieto, Alcaraz- } \\
\text { Rodríguez, Sánchez-Oliver } \\
\text { y Grimaldi-Puyanal (2018) }\end{array}$ & $P$ & 5o y 60 & - No se ha llevado a cabo la propuesta. \\
\hline $\begin{array}{l}\text { Morente-Oria, Romance- } \\
\text { García, Gil-Espinosa y } \\
\text { Benítez Porres (2018) }\end{array}$ & P & - & - No se ha llevado a cabo la propuesta. \\
\hline *P: propuesta; E: experincia; l: investigación &
\end{tabular}

*P: propuesta; E: experiencia; I: investigación

Tabla 2: Trabajos enfocados en Educación Secundaria Obligatoria (ESO) y Bachillerato.

\begin{tabular}{|c|c|c|c|}
\hline Autoría & Tipo* & $\begin{array}{l}\text { Etapa y } \\
\text { Curso }\end{array}$ & Resultados y/o conclusiones \\
\hline \multirow[t]{2}{*}{$\begin{array}{l}\text { Alcaraz, Sánchez y } \\
\text { Grimaldi (2018) }\end{array}$} & $P$ & ESO & - No se ha llevado a cabo la propuesta. \\
\hline & & & $\begin{array}{l}\text { - El alumnado valoró de forma muy positiva su participa- } \\
\text { ción, tanto al grado de satisfacción durante la situación } \\
(8,1) \text {, como por al grado de utilidad (8). }\end{array}$ \\
\hline \multirow[t]{2}{*}{ Almirall (2016) } & $\mathrm{E}$ & ESO: 1은 & $\begin{array}{l}\text { - El alumnado consolidó los principales objetivos de apren- } \\
\text { dizaje. }\end{array}$ \\
\hline & & & $\begin{array}{l}\text { - Los resultados obtenidos reforzaron la idea del potencial } \\
\text { de la gamificación como estrategia metodológica en el ám- } \\
\text { bito educativo y en la materia de EF. }\end{array}$ \\
\hline Cruz (2015) & $P$ & $\begin{array}{c}\text { ESO: } 3 \circ y \\
4 \stackrel{9}{-}\end{array}$ & - No se ha llevado a cabo la propuesta. \\
\hline
\end{tabular}

- El alumnado, al igual que el profesorado, valoraron de positivamente el uso de la gamificación como estrategia moti-

González, Zurita, Monguillot y vacional para el aprendizaje. La gran mayoría afirmó haber Almirall (2015) aprendido a aplicar la frecuencia cardíaca saludable.

- Uno de los aspectos que mejor funcionó fue la personalización de los retos, permitió atender a los diferentes ritmos de aprendizaje.

- Se comprobó que realizaron actividad física fuera del horario escolar, también con sus familiares más cercanos, fortaleciendo los vínculos parentales y además, indicaron haberse divertido con la dinámica de las actividades.

Martínez-Téllez, y Mora-González (2015)
ESO: 1ำ y - Con la consecución de los retos se consiguieron los obje4․ tivos, como el desarrollo de la creatividad del alumnado, la Bach.: 1ㅇ utilización de las TICs para hacer montajes, enviar correos, el uso de un lenguaje correcto.

- Se obtuvo la creencia de que con el uso de redes sociales se puede crear de forma potencial adherencia a la actividad física. 
- La gamificación aparece como una potente herramienta para movilizar conocimientos y consolidar aprendizajes. Los retos de diferente dificultad despertaron la curiosidad, sorpresa e intriga en el alumnado atendiendo a la vez a los diversos ritmos de aprendizaje. $Y$ todo ello, bajo una perspectiva lúdica, divertida y entretenida.

- La consecución de retos gamificados aumentó el compro-

Monguillot et al. (2015)

Mora-González y Martínez-Téllez (2015)
ESO: 2은 ESO: 1 으

$40 \quad$ - En cuanto a la valoración que el alumnado hizo sobre la E

Bach.: 1으 positiva aunque con ciertos aspectos a mejorar, como la explicación de las actividades.

- Al basar las clases en la diversión y en el disfrute del alumnado se favorecen aspectos como el trabajo en equipo, la motivación, la cooperación y la adquisición de valores. A su vez, favorece la disposición que muestra el alumnado a aprender.

Morente-Oria et al. (2018)

$P$

- No se ha llevado a cabo la propuesta.

- Se mejoró la motivación del alumnado tanto por la materia como por la práctica regular de actividad física involucrando a familiares, amigos y resto de la comunidad educativa. También provocó un interés extra del alumnado por asistir a clase.

Navarro et al. (2017)

$E$

ESO: 3‥ - Los alumnos pudieron disfrutar de variedad de actividades físico-deportivas y ello hizo posible reducir los conflictos

Bach.: 10 durante este periodo, mejorando la convivencia del centro.

- Los docentes involucrados consideran haber mejorado las competencias profesionales especialmente en la actualización didáctica, pedagógica y digital.

- El uso de metodologías activas en clase favoreció la creación de un clima de aula distendido. 
- La introducción de las nuevas tecnologías puede favorecer o reforzar el desarrollo de determinados contenidos incrementando el nivel de motivación y diversión del alumnado. Además, la utilización de las nuevas tecnologías favoreció Ortí (2018) E ESO el desarrollo de la competencia digital.

- Kahoot, aunque habitualmente es una herramienta utilizada para trabajar los contenidos teóricos, también presenta amplias posibilidades para desarrollar la motricidad del alumnado.

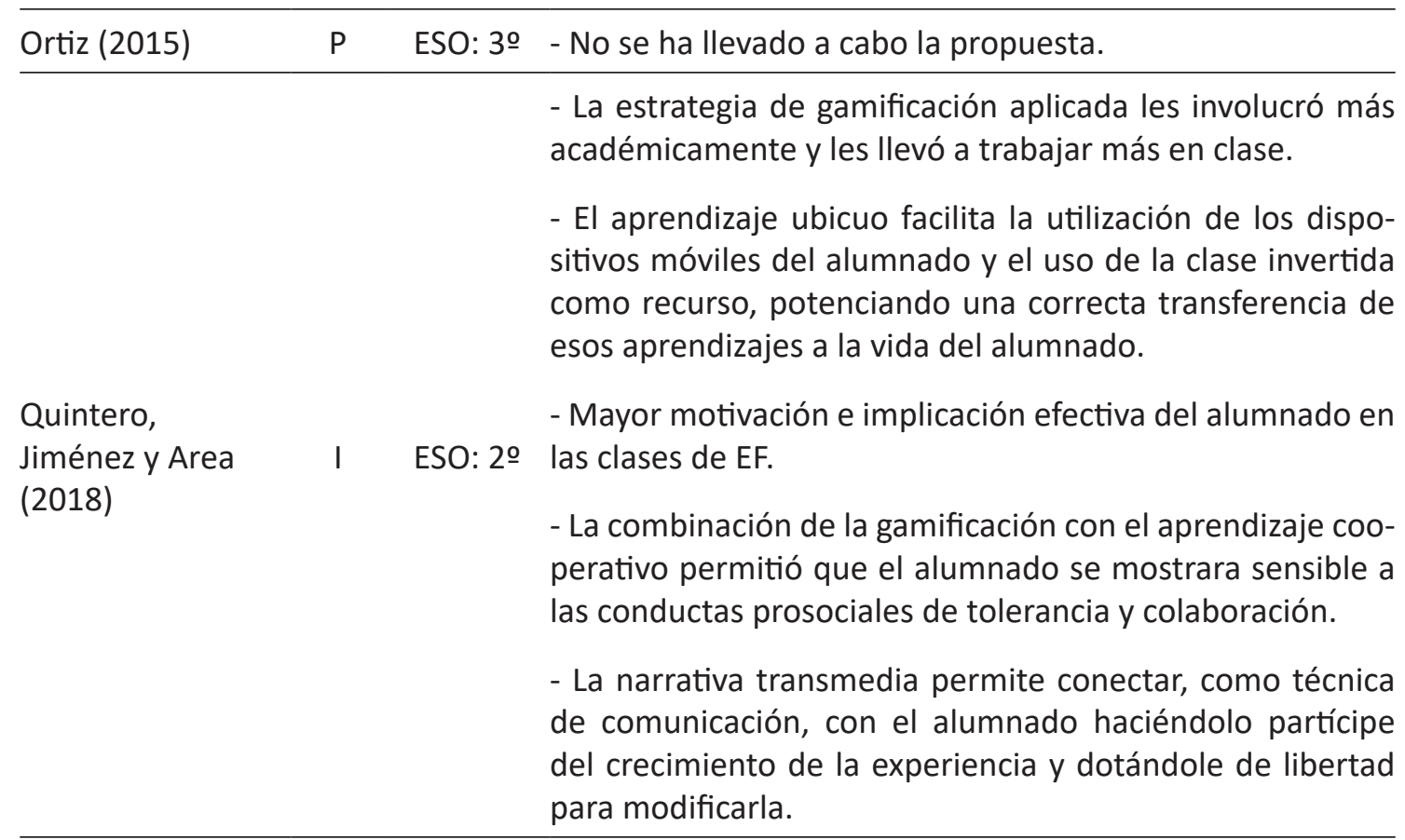

Tabla 3: Trabajos enfocados en el ámbito universitario.

\begin{tabular}{|c|c|c|c|}
\hline Autoría & Tipo* & Curso** & Resultados y/o conclusiones \\
\hline \multirow{3}{*}{$\begin{array}{l}\text { Caballero, } \\
\text { Domínguez, } \\
\text { Miranda y } \\
\text { Velo (2018) }\end{array}$} & \multirow{3}{*}{$\mathrm{E}$} & \multirow{3}{*}{$\begin{array}{l}\text { Grado en } \\
\text { Educación } \\
\text { Primaria }\end{array}$} & $\begin{array}{l}\text { - Fueron capaces de entender los diferentes tiempos orga- } \\
\text { nizativos y acciones asociadas, los distintos roles necesarios } \\
\text { para que funcionase, la necesidad de establecer una buena } \\
\text { comunicación, la asunción de responsabilidad, etc. }\end{array}$ \\
\hline & & & $\begin{array}{l}\text { - El mayor aprendizaje se debió a la interacción directa con } \\
\text { los/as niños/as del colegio, que les permitió darse cuenta de } \\
\text { la importancia de la actitud del docente, de la motivación que } \\
\text { despierta la incorporación de un hilo conductor a una activi- } \\
\text { dad, de la necesidad de realizar una reflexión final, etc. }\end{array}$ \\
\hline & & & $\begin{array}{l}\text { - El trabajo en equipo y la asunción de responsabilidades por } \\
\text { parte del alumnado universitario han sido los retos más difíci- } \\
\text { les de conseguir por el/la profesor/a. }\end{array}$ \\
\hline
\end{tabular}


García-

Fernández,

Fernández-

Gavira, Sán-

chez-Oliver y

Grado en
CAFD y ADE

Grimaldi-Pu-

yana (2017)

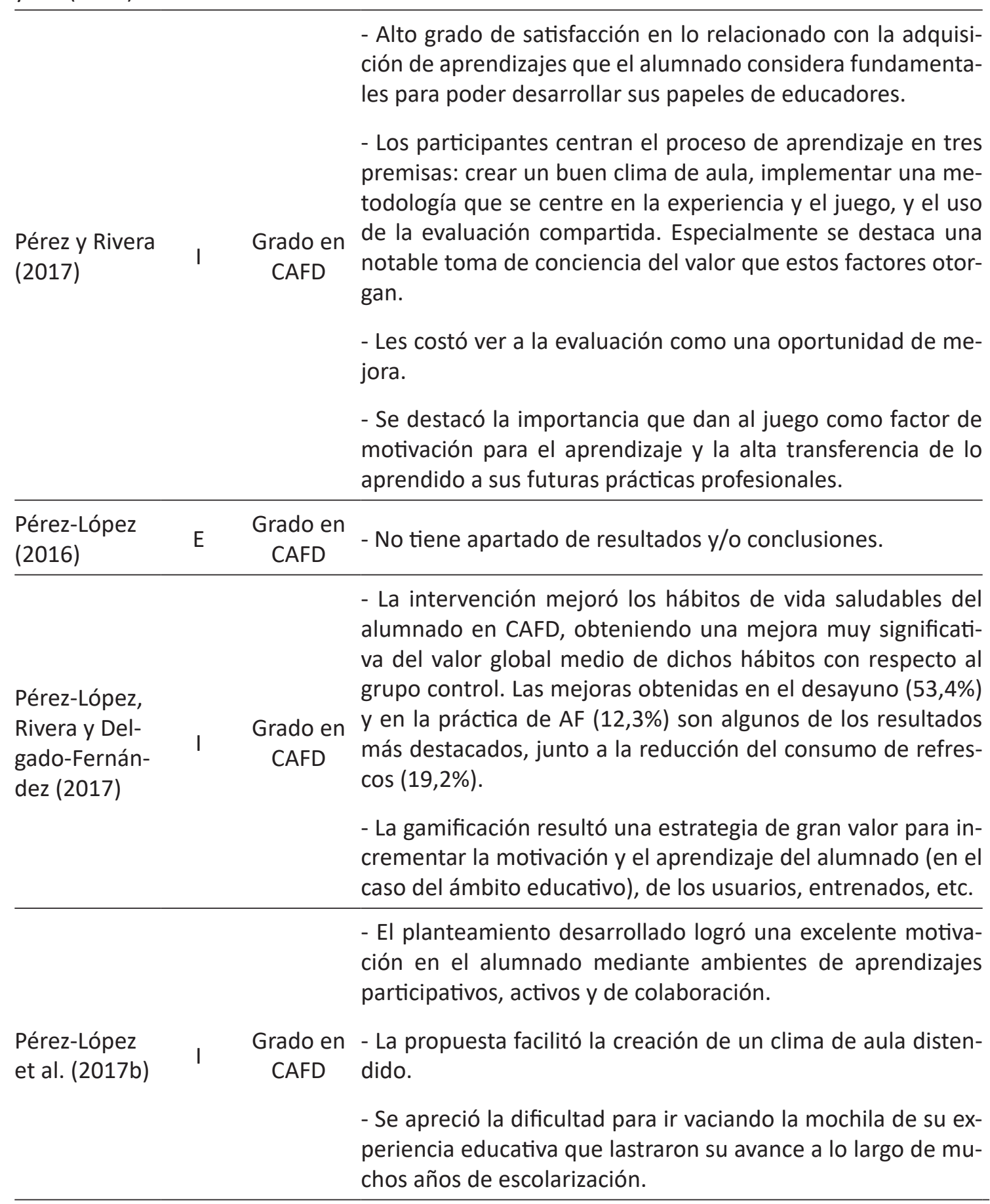

*P: propuesta; E: experiencia; I: investigación/ **CAFD: Ciencias de las Actividad Física y del Deporte; ADE: Administración y Dirección de Empresas. 


\section{DISCUSIÓN}

Son numerosos las conclusiones y resultados comunes que se obtuvieron tras el análisis de los trabajos incluidos en esta revisión. La consolidación de los objetivos principales y el aprendizaje significativo son de los más repetidos en los trabajos de gamificación en EF (Almirall 2016; González et al., 2015; MartínezTéllez et al., 2015; Monguillot et al., 2015; Ortí, 2018; Quintero et al., 2018). Asimismo, otros autores llegaron a dichas conclusiones tras la aplicación de trabajo de gamificación en otros contextos y/o asignaturas (Díaz y Troyano, 2013; Landers y Callan, 2011; Ordiz, 2017).

Se destaca de forma similar la potenciación de la motivación y de la satisfacción del alumnado (González et al., 2015; Navarro et al., 2017; MoraGonzález y Martínez-Téllez, 2015; Pérez y Rivera, 2017; Pérez-López et al., 2017a; Piña et al., 2015; Quintero et al., 2018) lo cual es común en el uso de esta estrategia como indicaron en diferentes trabajos (Díaz y Troyano, 2013; Landers y Callan, 2011; Ordiz, 2017; Ruizalba, Navarro y Jiménez, 2013; Estanyol, Montaña y Lalueza, 2013). La participación, trabajo en equipo y diversión también se vieron, como resultado, aumentadas entre estos trabajos (Almirall, 2016; Caballero et al., 2018; Monguillot et al., 2015; Mora-González y Martínez-Téllez, 2015; Navarro et al., 2017; Ortí, 2018; Quintero et al., 2018), así como el aprendizaje a diferentes ritmos siendo más personalizado (González et al., 2015; Monguillot et al., 2015), llevando incluso a conseguir que tanto el resto del centro como los familiares se involucren en el proceso de aprendizaje (Martínez-Téllez, et al., 2015; Mora-González y Martínez-Téllez, 2015; Navarro et al., 2017). Un ejemplo de esto se encuentra en el trabajo de Piña et al. (2015) con alumnos universitarios de Ingeniería donde un $65 \%$ consideraron haberse divertido como efecto principal y que esto llevaba a un ambiente en clase distendido y motivador, sin embargo apuntaron como negativo que las pruebas de evaluación pueden no considerarse como algo serio, e incluso que no se produzca ningún aprendizaje. Sin embargo, Escutia y Pamplona (2017) en su gamificación dentro de la asignatura de Derecho Romano, además de obtener las conclusiones positivas dichas anteriormente, sí señalaron la mejora del aprendizaje.

Es destacable cómo a través de la gamificación en las clases de EF se vio una mejora en la competencia en nuevas tecnologías como subrayaron MartínezTéllez et al., (2015), Monguillot et al., (2015), Ortí (2018) y Quintero et al. (2018) y en la adherencia a la EF fuera del horario escolar según Martínez-Téllez, et al. (2015), Monguillot et al. (2015), Navarro et al. (2017) y Pérez-López et al. (2017a), siendo en numerosos casos las nuevas tecnologías en sí usadas como parte protagonista de la gamificación como por ejemplo, indicaron Rodríguez, Loro y Villén (2015) tras su aplicación en sociología con alumnado de Magisterio. Por tanto, de forma general se puede considerar que se refuerza la idea de considerar la gamificación como una estrategia metodológica en el ámbito educativo en general y en la EF en particular (Almirall, 2016; Navarro et al., 2017; Pérez y Rivera, 2017; Pérez-López et al., 2017b). 


\section{CONCLUSIONES}

Este trabajo presenta una recopilación de los artículos existentes hasta la actualidad en diversas bases de datos, dónde la gamificación es integrada en el área de Educación Física. Los resultados muestran la reciente incorporación de esta metodología en este ámbito, encontrando los primeros trabajos publicados en 2015 y como han ido surgiendo más, encontrando un total de 19 artículos actualmente. Dato positivo, ya que, posiblemente, cada vez más docentes e investigadores son conscientes de los beneficios de la gamificación como herramienta educativa y más concretamente en EF, como se han presentado en el trabajo a partir de los artículos analizados. A pesar de ello, teniendo en cuenta las etapas educativas, se observa cómo Primaria y Bachillerato son en las que, considerablemente, menos trabajos existen publicados, encontrando E.S.O. principalmente presente. Estos datos dan pie a un claro llamamiento hacia el aumento de investigaciones y/o experiencias hacia estas etapas con presencia minoritaria de la gamificación, pero por supuesto, sin dejar de lado el resto de metodologías y estrategias con el fin de seguir avanzando y mejorando la enseñanza.

Por último, destacar que la recopilación de artículos en este trabajo, lo convierte en un útil recurso para docentes e investigadores/as pertenecientes al área de EF, ya que pueden acceder de forma sencilla a todos los artículos existentes hasta el momento, algo además favorable para los profesionales que quieran iniciarse en esta metodología, complementándose con la Guía de iniciación a la gamificación en Educación Física (Coterón, González, Mora y Fernández-Caballero, 2017).

\section{REFERENCIAS BIBLIOGRÁFICAS}

Alcaraz, V., Sánchez, A. J., y Grimaldi, M. (2018). El gran juego salvando a gea: gamificación y nuevas tecnologías en actividades físicas en medio natural. EmásF, Revista Digital de Educación Física, 9(54), 148-158. Recuperado de https://emasf. webcindario.com/El_gran_juego_\%20salvando_a_Gea_gamificacion_en_el medio natural.pdf

Almirall, L. (2016). Epic clans. Gamificando la Educación Física. Revista Tándem, (51), 67-73. Recuperado de https://www.grao.com/es/producto/epic-clans-gamificando-laeducacion-fisica

Caballero, P., Domínguez, G., Miranda, M. J., y Velo, C. (2018). Jornada de aventura "Superheroes en la ecoescuela": Una experiencia de aprendizaje servicio para conectar la universidad y el colegio. EmásF, Revista Digital de Educación Física, 9(54), 114-123. Recuperado de https://emasf.webcindario.com/Jornada_de aventura_superheroes en la ecoescuela.pdf

Coterón, J., González, J., Mora, C., y Fernández-Caballero, J. (2017). Guía de iniciación a la gamificación en Educación Física. Madrid, España: Fundación General de la Universidad Politécnica de Madrid. Recuperado de http://afipe.es/ assets/gu\%C3\%ADa-de-iniciaci $\%$ C3\%B3n-a-la-gamificaci\%C3\%B3n-en-educaci\%C3\%B3n-f\%C3\%ADsica.pdf 
Cruz, F. J. (2015). La conquista de Kömoda. Habilidad Motriz, (44), 4-20. Recuperado de https://docs.wixstatic.com/ugd/28d333 934300669e7e4a809baa9c08d57f7a23.pdf

Deterding, S., Khaled, R., Nacke, L. E., y Dixon, D. (2011). Gamification: Toward a definition. En Proceedings of the 2011 Workshop Gamification: Using Game Design Elements in Non-Game Contexts. Nueva York, NY: ACM. Recuperado de http:// gamification-research.org/wp-content/uploads/2011/04/02-Deterding-Khaled-NackeDixon.pdf

Díaz, J., y Troyano, Y. (2013). El potencial de la gamificación aplicado al ámbito educativo. En III Jornadas de Innovación Docente. Innovación Educativa: respuesta en tiempos de incertidumbre. Sevilla, España: Universidad de Sevilla. Recuperado de https://idus.us.es/xmlui/handle/11441/59067

Estanyol, E., Montaña, M., y Lalueza, F. (2013). Comunicar jugando. Gamificación en publicidad y relaciones públicas. En VII Internaional Conference on Communication and Reality: España, Barcelona: Facultat de Comunicació i Relacions Internacionals Blanquerna.

Escutia, R y Pamplona, S (2017). Gamificación en la asignatura Derecho Romano: un estudio de caso. En IV Congreso Internacional sobre Aprendizaje, Innovación y Competitividad, España, Zaragoza. Doi: 10.26754/CINAIC.2017.000001_091

Fernandez-Río, J., Calderón, A., Hortigüela, D., Pérez-Pueyo, Á., y Aznar, M. (2016). Modelos pedagógicos en educación física: consideraciones teórico-prácticas para docentes. Revista Española de Educación Física y Deportes, (413), 55-75. Recuperado de http://www.reefd.es/index.php/reefd/article/view/425

Fernández, J., Prieto, E., Alcaraz-Rodríguez, V., Sánchez-Oliver, A. J., y Grimaldi-Puyanal, M. (2018). Aprendizajes significativos mediante la Gamificación a partir del Juego de Rol: "Las Aldeas de la Historia". Espiral. Cuadernos del Profesorado, 11(22), 69-78. Doi: http://dx.doi.org/10.25115/ecp.v11i21

Game Marketing (2012). Estudio 2012. Gamificación, expectativas y grado de adopción en España. Recuperado de http://www.gamkt.com/estudio-2012/Estudio-2012-Gamificacion-Spanish-Version.pdf

García-Fernández, J., Fernández-Gavira, J., Sánchez-Oliver, A. J., y Grimaldi-Puyana, M. (2017). Gamificación y aplicaciones móviles para emprender: una propuesta educativa en la enseñanza superior. International Journal of Educational Research and Innovation (IJERI), (8), 248-259. Recuperado de https://www.upo.es/revistas/index. php/IJERI/article/view/2434

González, C., Zurita, C., Monguillot, M., y Almirall, L. (2015). Experiencias colaborativas para desarrollar hábitos saludables en Educación Física. Revista Tándem, (48), 3745. Recuperado de https://www.grao.com/es/producto/experiencias-colaborativaspara-desarrollar-habitos-saludables-en-educacion-fisica

González, C. S., y Mora, A. (2015). Técnicas de gamificación aplicadas en la docencia de Ingeniería Informática. ReVisión, 8 (1), 29-40. Recuperado de http://www. aenui.net/ojs/index.php?journal=revision\&page=article\&op=viewArticle\&path $\% 5 \mathrm{~B} \%$ $\underline{5 D}=152$

Guevara, J. M. (2015). Press Start, los videojuegos como recurso educativo: una propuesta de trabajo con Minecraft y Ciencias Sociales. Ar@cne. Revista Electrónica de Recursos en Internet sobre Geografía y Ciencias Sociales, (200), 1-15. Recuperado de http://www.ub.edu/geocrit/aracne/aracne-200.pdf 
Hernández, R., Fernández, C., y Baptista, P. (2010). Metodología de la investigación. Quinta edición. México: Mcgraw-Hill Interamericana Editores. Recuperado de https:// www.esup.edu.pe/descargas/dep investigacion/Metodologia $\% 20$ de $\% 201 a \% 20$ investigaci\%C3\%B3n\%205ta\%20Edici\%C3\%B3n.pdf

Johnson, D., Deterding, S., Kuhn, K. A., Staneva, A., Stoyanov, S., y Hides, L. (2016). Gamification for health and wellbeing: A systematic review of the literature. Internet Interventions, (6), 89-106. Doi: https://doi.org/10.1016/j.invent.2016.10.002

Kasurinen, J., y Knutas, A. (2016). Publication trends in gamification : A systematic mapping study Publication trends in gamification : A systematic mapping study. Computer Science Review, (27), 33-44. Doi: https://doi.org/10.1016/j.cosrev.2017.10.003

Landers, R. N., y Callan, R. C. (2011). Casual Social Games as Serious Games: The Psychology of Gamification in Undergraduate Education and Employee Training. En Serious Games and Edutainment Applications (pp. 399-423). London: Springer London. Doi. https://doi.org/10.1007/978-1-4471-2161-9 20

Lozada-Ávila, C., y Betancur, S. (2016). La gamificación en la educación superior: una revisión sistemática. Revista Ingenierías Universidad de Medellín, 16(31), 97-124. Doi: https://doi.org/10.22395/rium.v16n31a5

Marí, H. (2015). Estudio del aspecto motivador de la gamificación de los ejercicios de matemáticas y lengua castellana en el primer ciclo de primaria del "colegio bilingüe la devesa carlet" curso 2014 - 2015 (Trabajo Fin de Grado). Universidad Internacional de la Rioja, Valencia, España. Recuperado de https://reunir.unir. net/bitstream/handle/123456789/3412/MARI\%20ARNANDIS\%2c\%20HECTOR. pdf?sequence=1\&isAllowed $=y$

Marín, I., e Hierro, E. (2013). Gamificación : el poder del juego en la gestión empresarial y la conexión con los clientes. Madrid, España: Empresa Activa.

Martínez-Téllez, B., y Mora-González, J. (2015). Los Juegos de Rojas: el valor del reto y las TICS para crear adherencia a la actividad física fuera del aula. Habilidad Motriz, (44), 46-56. Recuperado de https://docs.wixstatic.com/ugd/28d333 934300669e7e4 a809baa9c08d57f7a23.pdf

Monguillot, M., González, C., Zurtia, C., Almirall, L., y Guitert, M. (2015). Play the Game: gamificación y hábitos saludables en Educación Física. Apunts. Educación Física, (119), 71-79. Doi: http://dx.doi.org/10.5672/apunts.2014-0983.es.(2015/1).119.04

Mora-González, J., y Martínez-Téllez, B. (2015). Los Juegos del Rojas: el aula en llamas. Habilidad Motriz, (44), 32-45. Recuperado de https://docs.wixstatic.com/ugd/2 8d333 934300669e7e4a809baa9c08d57f7a23.pdf

Morente-Oria, H., Romance-García, A. R., Gil-Espinosa, F. J., y Benítez-Porres, J. (2018). Programa de entrenamiento escolar gamificado para fomentar la actividad física saludable a través de una metodología innovadora de realidad aumentada (RA). Trances, 10(supl.1), 475-486. Recuperado de http://www.trances.es/papers/ TCS_10_supl01_09.pdf

Navarro, D., Martínez, R. e Pérez, I. J. (2017). El enigma de las 3 efes: fortaleza, fidelidad y felicidad. Revista Española de Educación Física y Deportes, (419), 73-85. Recuperado de http://www.reefd.es/index.php/reefd/article/view/607/549

Ordiz, T. (2017). Gamificación: La vuelta al mundo en 80 días. Revista Infancia, Educación y Aprendizaje, 3(2), 397-403. https://doi.org/10.22370/ieya.2017.3.2.755 
Ortegón, M. E. (2016). Gamificación de las matemáticas en la enseñanza del valor posicional de cantidades (Trabajo Fin de Máster). Universidad Internacional de la Rioja, Cali, Colombia. Recuperado de https://reunir.unir.net/bitstream/handle/123456789/4704/ORTEGON\%20YA\%C3\%91EZ\%2c\%20MARTHA\%20EMILIA Censurado.pdf?sequence=1\&isAllowed=y

Ortí, J. (2018). La gamificación en Educación Física. Desarrollo de la condición física a través de Kahoot. Revista Tándem, (60), 58-61. Recuperado de https://www. grao.com/ca/producte/revista-tandem-060-abril-18-desmontando-estereotipos-degenero-en-educacion-fisica-ta060

Ortiz, H. (2015). Ponle un 10 a Homer. Habilidad Motriz, (44), 21-31. Recuperado de https://docs.wixstatic.com/ugd/28d333 934300669e7e4a809baa9c08d57f7a23.pdf

Pérez, I., y Rivera, E. (2017). Formar docentes, formar personas: analisis de los aprendizajes logrados por estudiantes universitarios desde una experiencia de gamificación. Signo y Pensamiento, 36(70), 112-129. Doi: 10.11144/Javeriana.syp36-70.fdfp

Pérez-López, I. J. (2016). No te la juegues con tu salud, gamifícala: "La amenaza de los Sedentaris". Habilidad Motriz, (46), 42-48. Recuperado de https://docs.wixstatic. com/ugd/28d333 5fcd3c0b7b0f4a41a41697fb142d28c3.pdf

Pérez-López, I. J., Rivera, E., y Delgado-Fernández, M. (2017a). Mejora de hábitos de vida saludables en alumnos universitarios mediante una propuesta de gamificación. Nutrición Hospitalaria, 34(4), 942-951. Doi: http://dx.doi.org/10.20960/nh.669

Pérez-López, I. J., Rivera, E., y Trigueros, C. (2017b). “La profecía de los elegidos”: un ejemplo de gamificación aplicado a la docencia universitaria. Revista Internacional de Medicina y Ciencias de la Actividad Física y el Deporte, 17(66), 243-260. Doi: https://doi.org/10.15366/rimcafd2017.66.003

Piña, A. J., Ceada, Y., Andújar, J. M., Irigoyen, E., Gómez, V., y Artaza, F. (2015). Una propuesta para la motivación del alumnado de ingeniería mediante técnicas de gamificación. En Actas de las XXXVI Jornadas de Automática, España, Bilbao: Comité Español de Automática. Recuperado de https://www.ehu.eus/documents/3444171/4484747/77.pdf

Rodríguez, F., Loro, F., y Villén, S. (2015). Experiencia de gamificación en alumnos de magisterio para la evaluación de la asignatura Sociología de la educación mediante el uso de la plataforma Kahoot. En 3rd International conference on innovation, documentation and teaching technologies, España, Valencia: Universitat Politècnica de València. Doi: http://dx.doi.org/10.4995/INNODOCT.2015.1103

Ruizalba, J. L., Navarro, F., y Jiménez, S. (2013). Gamificación como estrategia de marketing interno. Intangible capital, 9(4), 1113-1144. Recuperado de http://www.redalyc. org/pdf/549/54929516008.pdf

Quintanal, F. (2016). Gamificación y la Física-Química de Secundaria. Education in the Knowledge Society (EKS), 17(3), 13-28. Doi: https://doi.org/10.14201/ eks20161731328

Quintero, L. E., Jiménez, F., y Area, M. (2018). Más allá del libro de texto. La gamificación mediada con TIC como alternativa de innovación en Educación Física. Retos, (34), 343-348. Recuperado de https://recyt.fecyt.es/index.php/retos/article/view/65514

Sardi, L., Idri, A., y Fernández-Alemán, J. L. (2017). A systematic review of gamification in e-Health. Journal of Biomedical Informatics, (71), 31-48. Doi: https://doi. org/10.1016/J.JBI.2017.05.011 